\title{
Pneumonia risk in COPD patients receiving inhaled corticosteroids alone or in combination: TORCH study results
}

\author{
C. Crim*, P.M.A. Calverley", J.A. Anderson`, B. Celli ${ }^{+}$, G.T. Ferguson ${ }^{\S}$, C. Jenkins ${ }^{f}$,

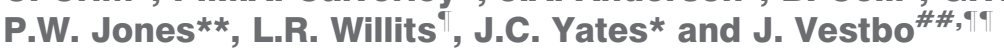

ABSTRACT: Inhaled corticosteroids (ICS) are important in reducing exacerbation frequency associated with chronic obstructive pulmonary disease (COPD). However, little is known about the risk of associated infections.

In a post hoc analysis of the TOwards a Revolution in COPD Health (TORCH) study, we analysed and identified potential risk factors for adverse event reports of pneumonia in this randomised, double-blind trial comparing twice-daily inhaled salmeterol (SAL) $50 \mu \mathrm{g}$, fluticasone propionate (FP) $500 \mu \mathrm{g}$, and the combination (SFC) with placebo in 6,184 patients with moderateto-severe COPD over 3 yrs.

Despite a higher withdrawal rate in the placebo arm, after adjusting for time on treatment, a greater rate of pneumonia was reported in the FP and SFC treatment arms (84 and 88 per 1,000 treatment-yrs, respectively) compared with SAL and placebo (52 and 52 per 1,000 treatmentyrs, respectively). Risk factors for pneumonia were age $\geqslant 55 \mathrm{yrs}$, forced expiratory volume in $1 \mathrm{~s}$ $<50 \%$ predicted, COPD exacerbations in the year prior to the study, worse Medical Research Council dyspnoea scores and body mass index $<25 \mathrm{~kg} \cdot \mathrm{m}^{-2}$. No increase in pneumonia deaths with SFC was observed; this could not be concluded for FP.

Despite the benefits of ICS-containing regimens in COPD management, healthcare providers should remain vigilant regarding the possible development of pneumonia as a complication in COPD patients receiving such therapies.

KEYWORDS: Chronic obstructive pulmonary disease, fluticasone propionate, inhaled corticosteroid, pneumonia, safety

hronic obstructive pulmonary disease (COPD) is associated with significant mortality and morbidity and patients with COPD are at increased risk of contracting pneumonia [1-3]. Conversely, COPD is the most common comorbid disease in patients hospitalised for community-acquired pneumonia $[1,2,4]$. Furthermore, patients with COPD who are hospitalised for pneumonia have been reported to exhibit higher mortality than patients without COPD [4-6].

Mechanisms to explain the increased risk for pneumonia in this population are not fully elucidated, but may in part be related to altered innate host mechanisms that result in increased carriage of potentially pathogenic microorganisms and altered function of immune effector cells [7-13]. Moreover, the carriage of such pathogenic organisms is related to disease severity, i.e. increased in patients with worse airflow obstruction (forced expiratory volume in $1 \mathrm{~s}(\mathrm{FEV} 1)<50 \%$ ) [9-14]. In addition, cross-sectional studies have shown increased microbiological colonisation of the lower respiratory tract during exacerbations of COPD compared with the stable state $[15,16]$.

Chronic colonisation with potentially pathogenic organisms can complicate ascertaining the aetiological microbe. Moreover, patients with more severe disease are often treated with inhaled and/or systemic corticosteroids in an attempt to either decrease the rate of exacerbations or provide symptomatic benefit. Although it is recognised that systemic corticosteroids may mask some of the clinical manifestations of pneumonia, such as fever, it is unclear to what extent inhaled corticosteroids (ICS) would also affect the clinical presentation.

The TOwards a Revolution in COPD Health (TORCH) study assessed the impact of the ICS
AFFILIATIONS

${ }^{*}$ GlaxoSmithKline, Research Triangle Park, NC,

${ }^{+}$Caritas-St Elizabeth's Medical

Center, Boston, MA, and

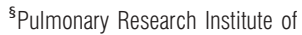

Southeast Michigan, Livonia, MI,

USA.

\#University Hospital Aintree,

Liverpool,

-GlaxoSmithKline, Middlesex

**University of London, London, and

"Wythenshawe Hospital,

Manchester, UK.

${ }^{f}$ Woolcock Institute of Medical

Research, Camperdown, Australia.

\#\#Hvidovre Hospital, Hvidovre,

Denmark.

CORRESPONDENCE

C. Crim

Medicine Development Centre -

Global Clinical Development

GlaxoSmithKline

Five Moore Drive

P.O. Box 13398

Research Triangle Park

NC 27709-3398

USA

E-mail: courtney.c.crim@gsk.com

Received:

Dec 222008

Accepted after revision:

April 262009

First published online:

May 142009 
fluticasone propionate (FP) in combination with the longacting $\beta$-agonist salmeterol (SAL) in reducing all-cause mortality [17]. It was observed in TORCH that despite a reduction in moderate and severe COPD exacerbations with FP either alone or as combination therapy (SFC), there was an increase in the probability of having a pneumonia reported as an adverse event (AE) compared with patients randomised to placebo or SAL. Similar findings were seen in a recent 2-yr study comparing SFC with tiotropium [18] and in a 1-yr prospective trial of SFC $50 \mu \mathrm{g} / 250 \mu \mathrm{g}$ versus SAL $50 \mu \mathrm{g}$ twice daily [19]. This analysis explores in more detail the increased pneumonia signal observed in the ICS-containing treatment arms in TORCH.

\section{METHODS}

\section{Study design}

The design of the TORCH trial has been previously described in detail in recent publications $[17,20]$. In brief, patients were randomised using a double-blind parallel group design to receive twice-daily administration of either FP $500 \mu \mathrm{g}$, SAL $50 \mu \mathrm{g}$, SFC (in a single device) $50 \mu \mathrm{g} / 500 \mu \mathrm{g}$, or placebo. Patients were followed for 3 yrs with the primary outcome being all-cause mortality. Secondary efficacy parameters included moderate (treatment with antibiotics and/or systemic corticosteroids) and/or severe (requiring hospitalisation) exacerbations and quality of life as measured by the St George's Respiratory Questionnaire.

\section{AEs and serious AEs}

To aid in the detection of AEs, at each study visit, after the patient was allowed to spontaneously mention any problems, the investigator asked the following standard questions: 1) "Have you had any (other) medical problems since your last visit/assessment?" and 2) "Have you taken any new medicines, other than those given to you within this study since your last visit/assessment?"' In TORCH, an AE was defined as any unfavourable and unintended sign (including a clinically significant abnormal laboratory finding), symptom or disease temporally associated with the use of the blinded study product, whether or not it was considered to be related to that product.

A serious $\mathrm{AE}$ (SAE) was any $\mathrm{AE}$ that resulted in any of the following outcomes: 1) death, 2) placing the patient in immediate risk of death (in the view of the investigator), 3) hospitalisation or prolonged existing hospitalisation, or 4) any other important medical event, which, based upon the medical judgment of the investigator, jeopardised the patient and may have required medical or surgical intervention to prevent one of the aforementioned outcomes. Once aware of an $\mathrm{SAE}$, the investigator was required to complete a separate case report form to provide all known information regarding the event. The form was updated when additional information was received. From these reports, narratives of the SAEs were compiled by GlaxoSmithKline's Global Clinical Safety and Pharmacovigilance department for subsequent review.

Patients who prematurely withdrew from the study were to have a follow-up visit 2 weeks after cessation of study drug where any AEs were recorded. Thus, the time period during which AEs and SAEs were collected and recorded started at the time the patient consented to participate in the study and ended at the end of the 2-week follow-up period. Patients were provided with a medication diary card in order to facilitate capture of this information.

\section{Collection of pneumonia events}

There were no protocol-defined criteria for pneumonia, nor were chest radiographs, sputum cultures or laboratory evaluations required to confirm the clinical diagnosis. In addition, no specific queries were made to the investigators with respect to pneumonia. Investigators recorded all AEs, including pneumonia reports, in the case report form. These events were subsequently coded using the Medical Dictionary for Regulatory Activities (MedDRA ${ }$, Version 8.1; International Federation of Pharmaceutical Manufacturers and Associations (IFPMA), Geneva, Switzerland). Events of physician-reported pneumonias were coded by MedDRA ${ }_{\AA}$ to the following terms and summarised to allow a more complete assessment of all physician-reported pneumonias: pneumonia, bronchopneumonia, lobar pneumonia, lung infection, pneumonia bacterial, pneumonia chlamydial, pneumonia necrotising, pneumonia staphylococcal, pneumonia streptococcal, superinfection lung, pneumonitis, pneumonia primary atypical, bronchopneumopathy, lung infection pseudomonal, and Pneumocystis jiroveci pneumonia.

\section{SAEs of pneumonia and data extraction}

The narrative reports of pneumonia as a SAE were initially reviewed with particular attention to supportive, confirmatory information; specifically, attainment of a chest radiograph, unilateral versus bilateral infiltrates, measurement of the white blood cell count, mention of a febrile state, and microbiological assessment of sputum, blood or pleural fluid, if present. The narratives were subsequently reviewed by one of the authors (C. Crim) to determine whether any of the above diagnostic procedures may have been conducted, although not explicitly described. For example, if the narrative indicated that the patient was treated for pneumonia in the anterior right upper lobe segment, it was assumed that a chest radiograph had been obtained, although not explicitly described. Other notations were made as to the presence of concomitant disorders that may have accounted for the radiograph appearance (e.g. congestive heart failure or lung cancer). Hence, as all the AEs of pneumonia were not definitively adjudicated and/or distinguished from other potential concomitant pulmonary parenchymal disorders, they are described here as "reported pneumonia."

In a separate analysis, an independent clinical end-points committee (CEC), which remained blind to treatment allocation of the patients, adjudicated all deaths using all available information, including information from medical records, autopsy reports and death certificates [21]. For the CEC to ascribe a death as being related to pneumonia, radiographic evidence of an infiltrate had to be present. From the review of these data sources, deaths due to pneumonia were identified.

\section{Statistical analysis}

Patients were included in the analysis if they took at least one dose of study medication. They were analysed according to the treatment they took for the majority of the treatment period, 
which, apart from one exception, was the treatment to which they were randomised.

The number and proportion of patients who were reported as having any of the above grouped pneumonia terms as an AE or SAE that started between treatment start and stop date inclusive was summarised by treatment group. Due to differential treatment exposure between the treatment groups, the rate of pneumonia events per 1,000 treatment-yrs was calculated by dividing the number of AEs by the number of years patients were exposed to study treatment, then multiplying by 1,000 .

The time to first pneumonia was compared between treatment groups using Kaplan-Meier estimates and the log-rank test, stratified by smoking status. Kaplan-Meier cumulative incidence curves were also produced.

To identify risk factors for pneumonia, a Cox's proportional hazards model for time to first pneumonia was fitted, using covariates of smoking status, age, \% predicted FEV1 (in Global Initiative for Chronic Obstructive Lung Disease (GOLD) stages), sex, exacerbations in the year prior to the study, body mass index (BMI), Medical Research Council (MRC) dyspnoea score and treatment. Interactions of these factors with treatment were fitted in this model, one at a time, with all covariates present in the model. At $<0.05$, p-values were considered statistically significant.

\section{RESULTS}

\section{Patients}

Of the 8,554 patients recruited into the study, 6,184 were randomised into one of the four treatment arms. One patient was randomised to placebo but took FP for the majority of the treatment period and was therefore analysed with the FP group. The demographic and baseline characteristics of this "safety" population are shown in table 1 . The mean age was $65 \mathrm{yrs}, 76 \%$ were male, mean smoking history was 48 pack-yrs, and baseline post-bronchodilator FEV1 was $44 \%$ of predicted.

\begin{tabular}{lccccc} 
TABLE 1 & Baseline characteristics (safety population) & \\
& Placebo & $\mathbf{S A L}$ & $\mathbf{F P}$ & $\mathbf{S F C}$ & Total \\
& & $\mathbf{5 0} \boldsymbol{\mu g}$ & $\mathbf{5 0 0} \boldsymbol{\mu \mathbf { g }}$ & $\mathbf{5 0} \mathbf{\mu g} / \mathbf{5 0 0} \boldsymbol{\mu \mathbf { g }}$ & \\
\hline & & & & & \\
Patients $\mathbf{n}$ & 1544 & 1542 & 1552 & 1546 & 6184 \\
Age yrs & $65 \pm 8$ & $65 \pm 8$ & $65 \pm 8$ & $65 \pm 8$ & $65 \pm 8$ \\
Sex & & & & & \\
$\quad$ Male & $1175(76)$ & $1176(76)$ & $1169(75)$ & $1164(75)$ & $4684(76)$ \\
$\quad$ Female & $369(24)$ & $366(24)$ & $383(25)$ & $382(25)$ & $1500(24)$ \\
Smoking status & & & & & \\
$\quad$ Former & $880(57)$ & $885(57)$ & $886(57)$ & $879(57)$ & $3530(57)$ \\
$\quad$ Current & $664(43)$ & $657(43)$ & $666(43)$ & $667(43)$ & $2654(43)$ \\
Total pack-yrs & $49 \pm 27$ & $49 \pm 29$ & $49 \pm 27$ & $47 \pm 27$ & $49 \pm 28$ \\
Baseline FEV ${ }^{\#}$ & $44(13)$ & $44(13)$ & $45(13)$ & $45(14)$ & $44(13)$ \\
Reversibility \% & $10 \pm 11$ & $10 \pm 11$ & $10 \pm 11$ & $10 \pm 11$ & $10 \pm 11$ \\
\hline
\end{tabular}

Data are presented as mean \pm SD or $n(\%)$, unless otherwise stated. SAL: salmeterol; FP: fluticasone propionate; SFC: SAL plus FP combination; FEV1: forced expiratory volume in $1 \mathrm{~s} .{ }^{*}$ : data presented as value (\% predicted postbronchodilator)
The mean extent of exposure to study medication was higher in all active treatment groups compared with placebo, and was greatest in the group that took SFC (table 2). There was an $8 \%$ increase in exposure over placebo in both the FP and SAL groups, and a $13 \%$ increase in exposure in the patients randomised to SFC.

\section{Pneumonia reported as AEs}

By definition, pneumonia events were also classified as exacerbations; however, these pneumonia-related exacerbations only constituted $7 \%$ of total exacerbations and $\sim 20 \%$ of the severe exacerbations. During the course of the study, there were more patients who had an AE report of pneumonia on FP or SFC compared with those in the SAL and placebo groups (14 and $16 \%$ versus 11 and $9 \%$, respectively; table 2 ). When adjusted for time on treatment, the rate of these events remained higher in the FP and SFC groups (84 and 88 per 1,000 treatment-yrs, respectively) compared with the SAL and placebo groups (52 and 52 per 1,000 treatment-yrs, respectively). In addition, the proportion of patients with more than one pneumonia event reported in the placebo, SAL, FP and SFC groups were 1.6, 1.1, 3.2 and 3.5\%, respectively.

Kaplan-Meier probability estimates revealed that the time to first pneumonia was significantly shorter in both arms containing the ICS (fig. 1). The hazard ratio for SFC versus placebo was 1.64 (95\% CI 1.33-2.02), representing a $64 \%$ increase in the risk of pneumonia at any time within $3 \mathrm{yrs}$ (table 3), with a similar effect for FP. Using an analysis of rates, it is estimated there would be one extra case of pneumonia for every 31 patients receiving treatment with SFC over 1 yr.

\section{Pneumonia reported as SAEs}

A similar trend was also observed for pneumonias reported as SAEs; more patients receiving an ICS-containing regimen reported a SAE-designated pneumonia compared with either SAL or placebo (table 2). Moreover, the pneumonia rate remained higher in both the FP and SFC groups when adjusted for time on treatment. To observe one extra SAE of pneumonia following treatment with SFC over $1 \mathrm{yr}$, the "number-neededto-harm" (NNH) would be 47 .

Presumptive or definitive evidence of performed chest radiography was identified in $72 \%$ of the SAE-designated pneumonia narratives and were similar across the treatment groups (75, 66, 70 and $76 \%$ for placebo, SAL, FP and SFC, respectively). Parenchymal infiltrates were described in $81 \%$ of these cases; other concomitant disorders included lung neoplasia, congestive heart failure, pleural effusions, bronchiectasis and pneumothorax. In the cases where there was no evidence that chest radiology was performed, concomitant disorders included subsequently confirmed lung neoplasia, sepsis and myocardial infarction (with and without cardiogenic shock). Moreover, these associated disorders did not occur predominantly in any specific treatment group.

Presence of fever and/or leukocytosis was reported in low numbers in both the ICS-containing (11 FP and three SFC) and non-ICS-containing (two placebo and five SAL) regimens. Although attempts at microbiological identification were infrequently noted $(12 \%)$, the most common reported pathogens from either blood, sputum or via fibreoptic bronchoscopy 


\begin{tabular}{|c|c|c|c|c|}
\hline & Placebo & SAL $50 \mu \mathrm{g}$ & FP $500 \mu \mathrm{g}$ & SFC $50 \mu \mathrm{g} / 500 \mu \mathrm{g}$ \\
\hline Total treatment exposure yrs & 3278 & 3531 & 3555 & 3700 \\
\hline \multicolumn{5}{|l|}{ AEs } \\
\hline Event rate per 1000 treatment-yrs & 52 & 52 & 84 & 88 \\
\hline \multicolumn{5}{|l|}{ SAEs } \\
\hline Patients & 86 & 99 & 150 & 157 \\
\hline Events & 97 & 105 & 184 & 204 \\
\hline Event rate per 1000 treatment-yrs & 30 & 30 & 52 & 55 \\
\hline \multicolumn{5}{|l|}{ Fatal SAEs } \\
\hline
\end{tabular}

were Streptococcus pneumoniae, Haemophilus influenzae, Klebsiella pneumoniae and Pseudomonas aeruginosa. No opportunistic pathogens were reported as a SAE in any individual from any treatment group and patients with culture-unconfirmed, nonfatal pneumonia clinically responded to conventional antibiotics, further suggestive that these patients were not infected with an opportunistic pathogen. However, there was one non-SAE of Pneumocystis jiroveci pneumonia reported from a patient

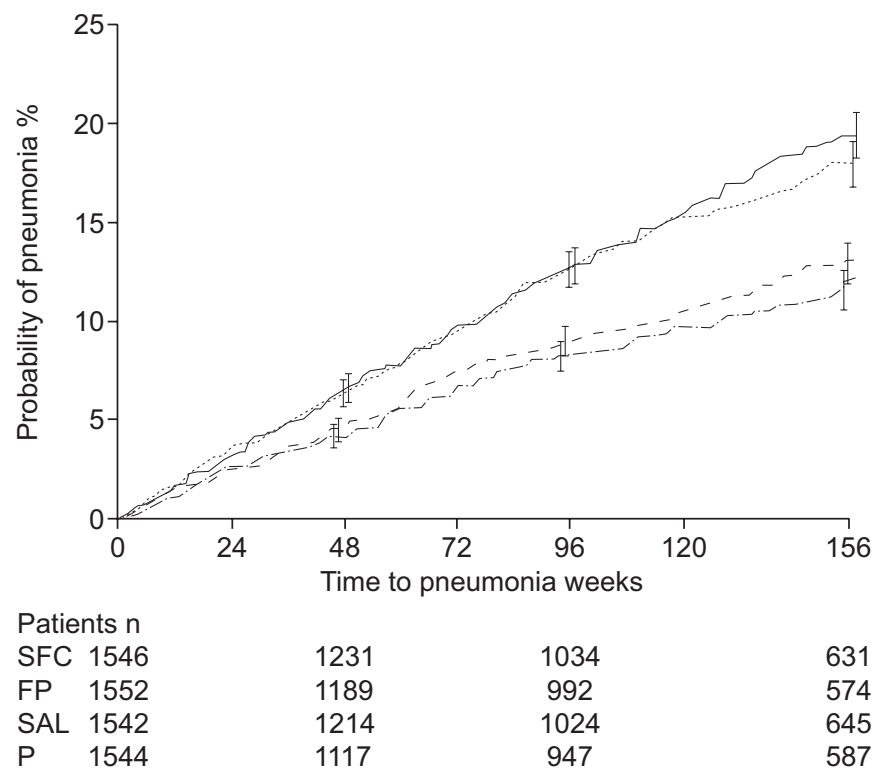

FIGURE 1. Kaplan-Meier estimate of time to first pneumonia for patients taking placebo $(\mathrm{P} ;-\cdot-\cdot-)$, salmeterol (SAL; - - - ), fluticasone propionate (FP; .....), and FP-SAL combination (SFC; - Values given below the figure are the numbers of patients at risk at randomisation and following 48,96 and 156 weeks of treatment. Vertical bars represent SE. randomised to placebo who was serologically positive for the HIV virus.

\section{Pneumonia deaths}

Deaths due to pneumonia as reported by the investigator occurred in $<1 \%$ of all patients (table 2 ). Based on these small numbers, no evidence was seen for a difference in any active treatment compared with placebo in the time to first fatal pneumonia event. As also shown in table 2, there was no increase in CEC-adjudicated deaths within the SFC arm compared with placebo and SAL, although there were six more deaths in the FP arm.

\section{Risk factors for pneumonia}

Irrespective of randomised treatment, the risk factors for pneumonia in this study population are summarised in figure 2. This shows the hazard ratios and associated 95\% confidence intervals for the factors from a Cox's proportional model for time to first pneumonia. These treatment-independent risk factors for pneumonia were older age (especially $>65$ yrs), lower $\%$ predicted FEV1 (especially $<30 \%$ predicted), any COPD exacerbations in the year prior to the study, worse MRC dyspnoea score (especially categories 4 and 5) and lower BMI. For example, patients with a FEV $1<30 \%$ predicted had a $72 \%$ increase in the risk of pneumonia compared with patients whose FEV1 was $\geqslant 50 \%$.

After adjusting for other risk factors, there was no evidence that current smokers were at greater risk than former smokers, or that sex was a risk factor. There was no interaction between age and ICS on the probability of pneumonia.

There was no evidence that these risk factors interacted with treatment, except for BMI $(p=0.032)$. Table 4 shows the Kaplan-Meier estimates of the probabilities of pneumonia for the four subgroups of BMI and their hazard ratios. It can be seen that the probabilities of pneumonia for ICS-containing arms versus non-ICS-containing arms were 17 versus $11 \%$ and 


\section{TABLE 3 Time to first pneumonia}

\begin{tabular}{|c|c|c|c|c|c|}
\hline Patients n & 1544 & 1542 & 1552 & 1546 & \\
\hline Probability ${ }^{\#}$ of pneumonia by 3 yrs $\%$ & 12.3 & 13.3 & 18.3 & 19.6 & \\
\hline Hazard ratio & & 1.09 & 1.53 & 1.64 & $1.52^{+}$ \\
\hline $95 \% \mathrm{Cl}^{\circ}$ & & $0.87-1.37$ & $1.24-1.89$ & $1.33-2.02$ & $1.32-1.76^{+}$ \\
\hline
\end{tabular}

SAL: salmeterol; FP: fluticasone propionate; SFC: SAL plus FP combination; ICS: inhaled corticosteroid (FP and SFC). ${ }^{*}$ : Kaplan-Meier estimate of probability; " : versus placebo unless otherwise stated; ${ }^{+}$: versus non-ICS (placebo and SAL).

13 versus $13 \%$, for BMI 25 to $<29 \mathrm{~kg} \cdot \mathrm{m}^{-2}$ and $\geqslant 29 \mathrm{~kg} \cdot \mathrm{m}^{-2}$, respectively. However, the corresponding probabilities were 25 versus $20 \%$ and 22 versus $12 \%$ for BMI $<20 \mathrm{~kg} \cdot \mathrm{m}^{-2}$ and 20 to $<25 \mathrm{~kg} \cdot \mathrm{m}^{-2}$. Thus, it appears that patients with lower BMI were not only more at risk of pneumonia, but also that in this population the association of ICS usage and pneumonia appeared stronger.

\section{DISCUSSION}

Current guidelines for the management of COPD recommend adding ICS to long-acting bronchodilators for patients with severe disease and/or frequent exacerbations. This recommendation is substantiated by recent publications demonstrating that ICS/long-acting $\beta$-agonist combinations result in a decreased rate of COPD exacerbations, including this study $[17-19,22,23]$. However, this present analysis suggests that

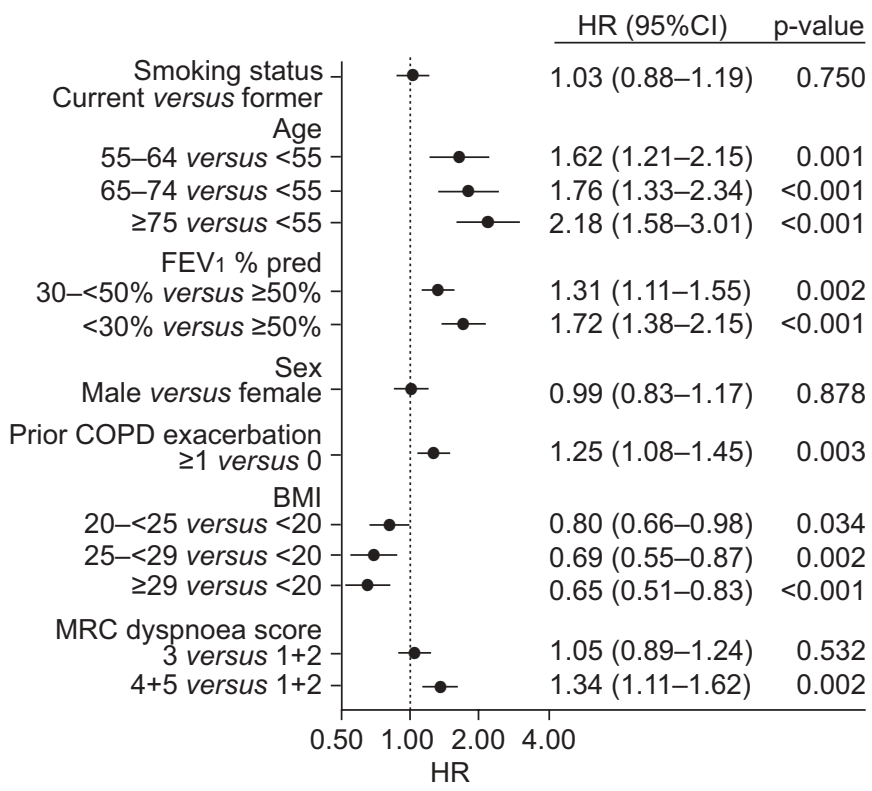

FIGURE 2. Risk factors for pneumonia: hazard ratios (HR) with $95 \% \mathrm{Cls}$ from Cox proportional hazards model using covariates of smoking status, age (in yrs), \% predicted forced expiratory volume in $1 \mathrm{~s}$ ( $F E V_{1}$; in Global Initiative for Chronic Obstructive Lung Disease (GOLD) stages), sex, chronic obstructive pulmonary disease (COPD) exacerbations in the year prior to the study, body mass index (BMI; in $\mathrm{kg} \cdot \mathrm{m}^{-2}$ ), Medical Research Council (MRC) dyspnoea score and treatment.
ICS-containing regimens are associated with increased reports of pneumonia. This observation is of concern in light of publications suggesting increased morbidity and mortality when pneumonia develops in patients with COPD [4-6, 24].

Patients receiving placebo withdrew at a significantly higher rate than those receiving active treatment, and the withdrawal rate was lowest for those in the SFC group [17]. To adjust for this differential drop-out, we also reported the pneumonia incidence rate as events per 1,000 treatment-yrs; the observation of higher pneumonia events still persisted in the ICScontaining regimens. In addition, the time to the first pneumonia event was considerably shorter in the ICScontaining-treatment arms and was clearly discernible at 6 months.

The increase in reports of pneumonia as an AE in the ICScontaining treatment arms occurred despite a decrease in overall COPD exacerbations, of which pneumonia was a subset. Signs and symptoms of COPD exacerbations and pneumonia often overlap [25] and it is possible that both overand under-diagnosis of pneumonia occurred, particularly as chest radiographs were not required to confirm the diagnosis. It is also noteworthy that the reports of pneumonia SAEs (i.e. death, hospitalisation or prolonged hospitalisation) as a percentage of the total pneumonias were no more frequent in the FP or SFC groups compared with placebo $(\sim 60 \%)$. This observation suggests that any episodes of pneumonia that occur with concomitant ICS treatment are unlikely to result in a more complex or complicated clinical course. In further support of this observation, no opportunistic pathogens were reported in any pneumonia episode; however, extensive microbiological investigations were not required or routinely undertaken in every case. Conversely, patients with COPD are chronically colonised with potential pathogens, which complicates ascertaining a specific aetiological agent using noninvasive techniques $[9,26,27]$ and also may not permit differentiation between exacerbation and pneumonia microbiologically.

Recently, ERNST et al. [24] reported the results of a nested casecontrol study within a cohort of nearly 176,000 patients with COPD that examined ICS use and the risk of hospitalisation for pneumonia. Compared with non-ICS users within the past year, patients receiving at least $1,000 \mu \mathrm{g} \cdot \mathrm{day}^{-1}$ of $\mathrm{FP}$ equivalent had a rate ratio for pneumonia hospitalisation of 2.25 (95\% CI 2.07-2.44). Conversely, the length of pneumonia 


\begin{tabular}{|c|c|c|c|c|c|}
\hline \multirow[t]{3}{*}{ TABLE 4} & \multicolumn{5}{|c|}{$\begin{array}{l}\text { Kaplan-Meier estimate of the impact of body mass index (BMI) on time to first pneumonia, comparing regimens with } \\
\text { and without inhaled corticosteroids (ICS) }{ }^{\#}\end{array}$} \\
\hline & & \multicolumn{4}{|c|}{ BMI $\mathbf{k g} \cdot \mathrm{m}^{-2}$} \\
\hline & & $<20$ & $20-<25$ & $25-<29$ & $\geqslant 29$ \\
\hline \multicolumn{6}{|l|}{ ICS } \\
\hline \multicolumn{6}{|l|}{ Non-ICS ${ }^{+}$} \\
\hline Patients $\mathrm{n}$ & & 407 & 1158 & 832 & 689 \\
\hline Probability & of event \% & $20(15-25)$ & $12(10-14)$ & $11(9-14)$ & $13(10-16)$ \\
\hline Hazard rat & & $1.30(1.02-1.64)$ & $1.98(1.67-2.34)$ & $1.60(1.30-1.98)$ & $1.00(0.79-1.26)$ \\
\hline
\end{tabular}

hospitalisation was similar whether or not patients were current users of ICS (mean 11.7 versus 11.8 days), as was all-cause mortality within 30 days of being hospitalised for pneumonia for patients dispensed ICS in the prior 2 months $(8.2 \%$ of 18,005 patients) compared with those who were not dispensed ICS (7.4\% of 5,937 patients) [24]. In TORCH, the mortality rate due to pneumonia in the SFC group was similar to both the placebo and SAL groups as reported by both the investigators and the CEC. Moreover, whereas the investigators may not have had confirmatory chest radiographs during their assessment, the CEC did have access to medical records and radiographs and required radiographic evidence of an infiltrate in order to adjudicate a death as due to pneumonia [21]. The number of ontreatment deaths attributable to pneumonia (as adjudicated by the CEC) was not different between the placebo and SFC groups. Although a mortality study, TORCH was not designed to evaluate pneumonia as a specific fatal event and, as such, was not adequately powered to detect differences in fatal pneumonias between treatment groups. However, these data, although limited by few events, suggest that any increase in pneumonia events with an ICS-containing COPD regimen does not lead to increased pneumonia mortality.

The mechanism/pathogenesis of increased pneumonia with ICS is unclear. It is generally recognised that exacerbations of COPD are frequently associated with infection. Thus, there is an apparent paradox whereby an ICS-containing regimen reduces the incidence of one infective complication of COPD (i.e. exacerbation) yet potentially increases another (i.e. pneumonia). It is possible that ICS can alter local immune mechanisms in the airways, as this class of compounds is quite effective in the management of the inflammatory aspects of asthma [28]. Conversely, there are no reports of excess pneumonia AEs with ICS use in randomised clinical trials in asthmatics. Although corticosteroids are felt to be less effective in modulating neutrophil function compared with other effector cells, such as eosinophils or mast cells [28], several reports suggest that ICScontaining regimens may reduce sputum neutrophilia in patients with COPD $[29,30]$, perhaps by decreasing neutrophil chemotaxis [31]. However, there is some evidence that SAL may alter neutrophil function either through decreased adhesion to airway epithelium [32] or capillary endothelium [33] or by inhibiting respiratory burst activity [34]; yet an increase in the pneumonia signal was not observed with SAL therapy in this trial. The observation of a pneumonia signal was unexpected and TORCH was not designed to address the mechanism. However, by multivariate analysis, risk factors for pneumonia regardless of treatment were advancing age $(\geqslant 55 \mathrm{yrs})$, poor lung function (FEV1 $<50 \%$ predicted), BMI $<25 \mathrm{~kg} \cdot \mathrm{m}^{-2}$ and a history of exacerbations in the year prior to study entry. We were unable to identify factors that further increased the risk for pneumonia in patients receiving a steroid-containing regimen. Although we have also provided a $\mathrm{NNH}$ to indicate the increased risk for pneumonia, it is important to note that $\mathrm{NNH}$, in contrast with the hazard ratio, depends on the underlying percentage of patients who have the event of concern and treatment duration. For example and as discussed, the reported pneumonia rates differed between individuals aged $\geqslant 55$ yrs and those younger; also, rates differed between those with an FEV $1<50 \%$ predicted and patients with less severe airflow obstruction. Thus, the NNH would differ between these different age groups (or groups based on lung function), simply due to the difference in the underlying rate; conversely, the actual increased risk due to SFC in each of these different groups was observed to be similar to the overall increase of $64 \%$.

Use of an ICS regimen does decrease the rate of exacerbations. Since the clinical presentation of COPD exacerbations and pneumonia often overlap, it remains critical for the healthcare professional to remain vigilant for the possible development of pneumonia and implement early and appropriate therapy.

\section{CLINICAL TRIALS}

This study is registered at Clinicaltrials.gov (NCT00268216).

\section{SUPPORT STATEMENT}

This study was funded by GlaxoSmithKline. Study number: SCO30003.

\section{STATEMENT OF INTEREST}

Statements of interest for all of the authors and the study itself can be found at www.erj.ersjournals.com $/ \mathrm{misc} /$ statements.dtl 


\section{ACKNOWLEDGEMENTS}

The authors acknowledge technical support from Gardiner-Caldwell Communications (Macclesfield, UK) in the preparation of this manuscript; this support was funded by GlaxoSmithKline.

\section{REFERENCES}

1 Farr BM, Bartlett CLR, Wadsworth J, et al. Risk factors for community-acquired pneumonia diagnosed upon hospital admission. British Thoracic Society Pneumonia Study Group. Respir Med 2000; 94: 954-963.

2 Lange P, Vestbo J, Nyboe J. Risk factors for death and hospitalisation from pneumonia. A prospective study of a general population. Eur Respir J 1995; 8: 1694-1698.

3 Fry AM, Shay DK, Holman RC, et al. Trends in hospitalizations for pneumonia among persons aged 65 years or older in the United States, 1988-2002. JAMA 2005; 294: 2712-2719.

4 Holguin F, Folch E, Redd SC, et al. Comorbidity and mortality in COPD-related hospitalizations in the United States, 1979 to 2001. Chest 2005; 128: 2005-2011.

5 Rello J, Rodriguez A, Torres A, et al. Implications of COPD in patients admitted to the intensive care unit by communityacquired pneumonia. Eur Respir J 2006; 27: 1210-1216.

6 Restrepo MI, Mortensen EM, Pugh JA, et al. COPD is associated with increased mortality in patients with community-acquired pneumonia. Eur Respir J 2006; 28: 346-351.

7 Nair MPN, Kronfol ZA, Schwartz SA. Effects of alcohol and nicotine on cytotoxic functions of human lymphocytes. Clin Immunol Immunopathol 1990; 54: 395-409.

8 Honda Y, Takahashi H, Kuroki Y, et al. Decreased contents of surfactant proteins $\mathrm{A}$ and $\mathrm{D}$ in BAL fluids of healthy smokers. Chest 1996; 109: 1006-1009.

9 Mobbs KJ, van Saene HKF, Sunderland D, et al. Oropharyngeal gram-negative bacillary carriage in chronic obstructive pulmonary disease: relation to severity of disease. Respir Med 1999; 93: 540-545.

10 Prieto A, Reyes E, Bernstein ED, et al. Defective natural killer and phagocytic activities in chronic obstructive pulmonary disease are restored by glycophosphopeptical (Inmunoferón). Am J Respir Crit Care Med 2001; 163: 1578-1583.

11 Hodge S, Hodge G, Scicchitano R, et al. Alveolar macrophages from subjects with chronic obstructive pulmonary disease are deficient in their ability to phagocytose apoptotic airway epithelial cells. Immunol Cell Biol 2003; 81: 289-296.

12 Naylor EJ, Bakstad D, Biffen M, et al. Haemophilus influenzae induces neutrophil necrosis: a role in chronic obstructive pulmonary disease? Am J Respir Cell Mol Biol 2007; 37: 135-143.

13 Yoshikawa T, Dent G, Ward J, et al. Impaired neutrophil chemotaxis in chronic obstructive pulmonary disease. Am J Respir Crit Care Med 2007; 175: 473-479.

14 Wilkinson TMA, Patel IS, Wilks M, et al. Airway bacterial load and FEV1 decline in patients with chronic obstructive pulmonary disease. Am J Respir Crit Care Med 2003; 167: 1090-1095.

15 Monsó E, Ruiz J, Rosell A, et al. Bacterial infection in chronic obstructive pulmonary disease. A study of stable and exacerbated outpatients using the protected specimen brush. Am J Respir Crit Care Med 1995; 152: 1316-1320.
16 Papi A, Bellettato $\mathrm{CM}$, Braccioni F, et al. Infections and airway inflammation in chronic obstructive pulmonary disease severe exacerbations. Am J Respir Crit Care Med 2006; 173: 1114-1121.

17 Calverley PMA, Anderson JA, Celli B, et al. Salmeterol and fluticasone propionate and survival in chronic obstructive pulmonary disease. $N$ Engl J Med 2007; 356: 775-789.

18 Wedzicha JA, Calverley PMA, Seemungal TA, et al. The prevention of chronic obstructive pulmonary disease exacerbations by salmeterol/fluticasone propionate or tiotropium bromide. Am J Respir Crit Care Med 2008; 177: 19-26.

19 Ferguson GT, Anzueto A, Fei R, et al. Effect of fluticasone propionate/salmeterol $(250 / 50 \mu \mathrm{g})$ or salmeterol $(50 \mu \mathrm{g})$ on COPD exacerbations. Respir Med 2008; 102: 1099-1108.

20 The TORCH Study Group. The TORCH (TOwards a Revolution in COPD Health) survival study protocol. Eur Respir J 2004; 24: 206-210.

21 McGarvey LP, John M, Anderson JA, et al. Ascertainment of causespecific mortality in COPD: operations of the TORCH Clinical Endpoint Committee. Thorax 2007; 62: 411-415.

22 Calverley PM, Boonsawat W, Cseke Z, et al. Maintenance therapy with budesonide and formoterol in chronic obstructive pulmonary disease. Eur Respir J 2003; 22: 912-919.

23 Calverley P, Pauwels R, Vestbo J, et al. Combined salmeterol and fluticasone in the treatment of chronic obstructive pulmonary disease: a randomised controlled trial. Lancet 2003; 361: 449-456.

24 Ernst P, Gonzalez AV, Brassard P, et al. Inhaled corticosteroid use in chronic obstructive pulmonary disease and the risk of hospitalization for pneumonia. Am J Respir Crit Care Med 2007; 176: $162-166$.

25 Lieberman D, Lieberman D, Gelfer Y, et al. Pneumonic vs nonpneumonic acute exacerbations of COPD. Chest 2002; 122: 1264-1270.

26 Sethi S, Maloney J, Grove L, et al. Airway inflammation and bronchial bacterial colonization in chronic obstructive pulmonary disease. Am J Respir Crit Care Med 2006; 173: 991-998.

27 Cabello H, Torres A, Celis R, et al. Bacterial colonization of distal airways in healthy subjects and chronic lung disease: a bronchoscopic study. Eur Respir J 1997; 10: 1137-1144.

28 Jeffery P. Anti-inflammatory effects of inhaled corticosteroids in chronic obstructive pulmonary disease: similarities and differences to asthma. Expert Opin Investig Drugs 2005; 14: 619-632.

29 Barnes NC, Qiu YS, Pavord ID, et al. Antiinflammatory effects of salmeterol/fluticasone propionate in chronic obstructive lung disease. Am J Respir Crit Care Med 2006; 173: 736-743.

30 Yildiz F, Kaur AC, Ilgazli A, et al. Inhaled corticosteroids may reduce neutrophilic inflammation in patients with stable chronic obstructive pulmonary disease. Respiration 2000; 67: 71-76.

31 Llewellyn-Jones CG, Hill SL, Stockley RA. Effect of fluticasone propionate on neutrophil chemotaxis, superoxide generation, and extracellular proteolytic activity in vitro. Thorax 1994; 49: 207-212.

32 Bloemen PGM, van den Tweel MC, Henricks PA, et al. Increased cAMP levels in stimulated neutrophils inhibit their adhesion to human bronchial epithelial cells. Am J Physiol 1997; 272: L580-L587.

33 Bolton PB, Lefevre P, McDonald DM. Salmeterol reduces earlyand late-phase plasma leakage and leukocyte adhesion in rat airways. Am J Respir Crit Care Med 1997; 155: 1428-1435.

34 Ottonello L, Morone $\mathrm{P}$, Dapino $\mathrm{P}$, et al. Inhibitory effect of salmeterol on the respiratory burst of adherent human neutrophils. Clin Exp Immunol 1996; 106: 97-102. 